\title{
3D face recognition algorithm of alignment and fitting (Retraction Notice)
}

\section{Yifei Wu, Yao Cheng, Nan Yang}

Yifei Wu, Yao Cheng, Nan Yang, "3D face recognition algorithm of alignment and fitting (Retraction Notice)," Proc. SPIE 9631, Seventh International Conference on Digital Image Processing (ICDIP 2015), 96311A (6 July 2015); doi: $10.1117 / 12.2197158$

Event: Seventh International Conference on Digital Image Processing (ICDIP15), 2015, Los Angeles, United States 


\section{D face recognition algorithm of alignment and fitting (retraction notice)}

Yifei Wu, Yao Cheng, Nan Yang

Dalian Air Force Communication NCO Academy (China)

Proc. SPIE 9631, Seventh International Conference on Digital Image Processing (ICDIP 2015), 96311A

(July 6, 2015); doi:10.1117/12.2197158

From Conference Volume 9631

Seventh International Conference on Digital Image Processing (ICDIP 2015)

Charles M. Falco; Xudong Jiang

Los Angeles, United States | April 09, 2015

Online Publication Date: July 6, 2015

Withdrawn from Publication: April 11, 2016

This paper was retracted from the SPIE Digital Library on April 11, 2016, by the publisher upon verification that substantial portions of the paper were copied from the following work without attribution or permission:

M.M.J. Gerlach and C.T. Rooijers, "3D Face Recognition: Data Processing: Registration and Deformation," Bachelor of Science Thesis, Delft University of Technology, September 7, 2013. 